\title{
Potential benefits of pro- and prebiotics on intestinal mucosal immunity and intestinal barrier in short bowel syndrome
}

\author{
Christos N. Stoidis ${ }^{1 *}$, Evangelos P. Misiakos ${ }^{2}$, Paul Patapis ${ }^{2}$, Constantine I. Fotiadis ${ }^{2}$ and \\ Basileios G. Spyropoulos ${ }^{3}$ \\ ${ }^{1} 1$ st Department of Surgery, Athens Navy Hospital, 70 Deinocratous Street, Athens, Greece \\ ${ }^{2} 3$ rd Department of Surgery, University of Athens School of Medicine, Attikon University Hospital, 1 Rimini Street, Chaidari, \\ Athens, Greece \\ ${ }^{3} 1$ st Department of Propaedeutic Surgery, University of Athens School of Medicine, Hippokration Hospital, Athens, Greece
}

\section{Abstract}

The mechanism of impaired gut barrier function in patients with short bowel syndrome (SBS) is poorly understood and includes decreased intestinal motility leading to bacterial overgrowth, a reduction in gut-associated lymphoid tissue following the loss of intestinal length, inhibition of mucosal immunity of the small intestine by intravenous total parental nutrition, and changes in intestinal permeability to macromolecules. Novel therapeutic strategies (i.e. nutritive and surgical) have been introduced in order to prevent the establishment or improve the outcome of this prevalent disease. Pre- and probiotics as a nutritive supplement are already known to be very active in the intestinal tract (mainly in the colon) by maintaining a healthy gut microflora and influencing metabolic, trophic and protective mechanisms, such as the production of SCFA which influence epithelial cell metabolism, turnover and apoptosis. Probiotics have been recommended for patients suffering from SBS in order to decrease bacterial overgrowth and prevent bacterial translocation, two major mechanisms in the pathogenesis of SBS. The present review discusses the research available in the international literature, clinical and experimental, regarding probiotic supplementation for this complicated group of patients based on the clinical spectrum and pathophysiological aspects of the syndrome. The clinical data that were collected for the purposes of the present review suggest that it is difficult to correctly characterise probiotics as a preventive or therapeutic measure. It is very challenging after all to examine the relationship of the bacterial flora, the intestinal barrier and the probiotics as, according to the latest knowledge, demonstrate an interesting interaction.

\section{Key words: Probiotics: Short bowel syndrome: Bacterial translocation: Intestinal barrier: Bacterial overgrowth}

Surgical therapy forms, nowadays, a major part of the treatment regimen in various human small-bowel abnormalities or malignancies, congenital or acquired, and is frequently applied, as well, in the palliative management of several other incurable diseases. The loss, however, of a significant amount of the functional and absorptive surface of the small intestine results in a malnutrition and malabsorption state described as short bowel syndrome (SBS) ${ }^{(1)}$.

Although the pathogenesis of SBS is not entirely clear, it is presumed to be an adaptation process in which a large number of cell types, interacting molecular signals, including cytokines and growth factors, and various molecules on the endothelial cell surface participate ${ }^{(2)}$. As a result, this syndrome is a complex interplay among a plethora of pathophysiological processes, including cellular apoptosis and proliferation, epithelial regeneration and neoangiogenesis, activation of the immune system, tissue structural remodelling and bacterial flora alteration ${ }^{(3)}$.
Accumulating evidence suggests that probiotics and prebiotics represent promising candidates for the prevention and control of several gastrointestinal disorders ${ }^{(4)}$. Recent experimental studies in animal models and clinical trials of patients with short bowel diseases have indicated that the oral administration of probiotics and prebiotics may effectively down-modulate the severity of bacterial translocation (BT), which seems to be the main pathophysiological aspect of $\operatorname{SBS}^{(5)}$.

Although there are fewer experimental data and limited experience in human trials, these studies provide supporting evidence for the potential protective effects of probiotics in induced intestinal injury, not only by surgical intervention and the underlying pathology but also from the use of total parenteral nutrition. While the mechanisms of SBS and pre-probiotic interactions continue to be under investigation, the hypothesis of an underlying

Abbreviations: BT, bacterial translocation; LGG, Lactobacillus rhamnosus GG; NEC, necrotising enterocolitis; PN, parenteral nutrition; SBS, short bowel syndrome.

*Corresponding author: Dr Christos N. Stoidis, fax+30 2310 643149, email aris.80@hotmail.com 
enhancement of cellular immunological defence should be better investigated.

With the present literature review based on an extended research of international data on the worldwide web we would like, first of all, to define the terms SBS, BT and syn-, pre- and probiotics, terms that are often used in paediatric and gastrointestinal surgery. In particular, we aim to emphasise that further investigation at the clinical and experimental level should be carried out in order to determine the exact role that probiotics play, protective or preventive, in SBS.

\section{Short bowel syndrome}

The critical reduction of functional gut mass below the minimal amount necessary for adequate digestion and absorption to satisfy body nutrient and fluid requirements for maintenance in adults and growth in children is characterised as intestinal failure ${ }^{(6)}$. Causes of intestinal failure include several diseases such as congenital intestinal atresia, aganglionosis and gastroschisis, neoplasms, inflammation, radiation enteritis, malrotation with midgut volvulus, mesenteric ischaemia and emboli, abdominal wall defects and necrotising enterocolitis (NEC) in neonates and children ${ }^{(7)}$. Massive resection of the small bowel results in a complex of symptoms and metabolic alterations $^{(8)}$. The pathophysiological process after intestinal failure in order to maintain intestinal recovery, nutrition and long-term survival describes SBS.

The implied difference between the two entities (i.e. intestinal failure and SBS) is that SBS is associated with significant loss of absorptive surface area, whereas intestinal failure is a lack of satisfactory absorption despite an intestinal surface area that should be capable of sustaining positive fluid and electrolyte and nutrient balance ${ }^{(9)}$. With the advent of new therapies on the horizon, there is more interest in differentiating the two entities, as some therapies may work for one and not the other. A patient with SBS may also have intestinal failure, whereas not all patients with intestinal failure have SBS.

Small-intestinal bacterial overgrowth seems to be the triggering phenomenon for SBS and may contribute to symptoms, mucosal injury and malnutrition ${ }^{(10)}$. Its occurrence relates to alterations in intestinal anatomy, motility and gastric acid secretion.

These alterations in intestinal anatomy during the surgical procedure and especially the remnant small bowel length, an intact ileocaecal valve, intestinal continuity and preservation of the colon are important factors for survi$\mathrm{val}^{(11)}$. The ability of the residual intestine to adapt after massive small bowel resection appears also of extreme importance $^{(12)}$. In this setting, adaptation means progressive recovery from intestinal failure throughout which the small bowel increases its absorptive surface area and its functional capacity in an attempt to meet the body's metabolic and growth needs. Intestinal adaptation constitutes the best option for patients with SBS. In humans, intestinal adaptation begins within $24-48 \mathrm{~h}$ of resection and includes morphological (structural adaptation) and functional (functional adaptation) changes of the residual bowel. Structural adaptation includes increasing bowel diameter and length, lengthening the villi, deepening the crypts, and increasing the rate of enterocyte proliferation, finally resulting in an increased absorptive surface area and an increased number of enterocytes. Functional adaptation entails modifications of the brush-border membrane permeability and up-regulation of carrier-mediated transport, ultimately resulting in increased nutrient absorption by isolated enterocytes. The dynamic process of enterocyte turnover is a function of the rates of crypt cell proliferation, migration along the crypt-villus axis, death via apoptosis ${ }^{(13)}$ and proliferation. A large body of work exists on the interactions between epithelial cell proliferation and capillary growth $^{(14)}$. Specifically in the intestine, angiogenic growth factor supplementation has been shown to enhance mucosal growth in transplanted intestinal grafts in rats ${ }^{(15)}$. A recent study ${ }^{(6)}$ demonstrated that vascular endothelial growth factor inhibition within the saliva resulted in decreased capillary growth and a decreased adaptive response after massive intestinal loss in a murine model ${ }^{(16)}$. Although the interaction between capillary growth and enterocyte proliferation during resectioninduced adaptation would appear to be obvious, the temporal profile of this growth in relation to the expression of specific genes and activity of discrete signalling cascades is presently unknown ${ }^{(17)}$.

Immunological alterations are also present during the above adaptation process. In a recent study it was demonstrated that surgical stress in a rat small-intestinal resection model decreases systemic $\mathrm{CD}_{4}{ }^{+} \mathrm{T}$ cells and suppresses Th1 cytokine production without increasing Th2 cytokines $^{(18)}$. These changes may affect host immune defence, and increase the incidence of postoperative infections, thus indicating that small-intestinal resection rats are useful not only as a malabsorption model, but also as a surgical stress model.

The pathophysiological process signalling SBS may also be affected by nutritional status, the route of feeding and the adequacy of specific nutrients in the diet. As the capacity for uptake of nutrients is strongly reduced after massive resection the major problem in management of patients with SBS is malnutrition ${ }^{(3)}$.

The management of patients with bacterial overgrowth, intestinal failure and SBS remains, for the most part, primarily empirical and comprises antibiotic therapy and correction of any associated nutritional deficiencies. Historically, mortality was nearly universal in patients with intestinal failure until the introduction of parenteral nutrition $(\mathrm{PN})$ around $1970^{(19)}$. PN has subsequently become the primary treatment for intestinal failure, but can be associated with severe life-threatening complications ${ }^{(20)}$. These complications lead to mortality in up to $30 \%$ of 
patients with intestinal failure overall and up to $50 \%$ in paediatric patients with intestinal failure ${ }^{(21)}$. These high mortality rates, most commonly as a result of severe or recurrent line-related sepsis, venous thrombosis leading to loss of vascular access or $\mathrm{PN}$-associated liver disease (PNALD), have been addressed with the introduction of successful intestinal transplantation ${ }^{(22)}$. With severe SBS, intestinal transplantation must be considered when no other surgical or medical measures are available ${ }^{(23)}$. Although patients' survival continues to improve after intestinal transplantation (currently reported as 80 and $54 \%$ at 1 and 5 years, respectively), pre-transplant mortality remains higher than that of any other group awaiting solidorgan transplantation $^{(24)}$. Innovative surgical, nutritive and pharmacological therapeutic measures in SBS patients are constantly changing the prognosis of this potentially devastating condition ${ }^{(25)}$. Parenteral strategies employed to minimise total parenteral nutrition damage include reducing glucose infusion rates, reducing parenteral protein load and reducing parenteral lipid load. Furthermore, preliminary studies suggest that fish oil-based lipid solutions may have a beneficial effect on PNALD. Ultimately, provision of enteral nutrition is imperative for preventing or reversing PNALD as well as facilitating bowel adaptation. While studies of trophic hormones are ongoing, the most reliable current method to facilitate adaptation is to provide enteral nutrition ${ }^{(3)}$. Continuous enteral feeding remains the mainstay of enteral nutrition support. Probiotics seem to be part of enteral nutrition and we will analyse those in the oncoming sections along with their possible effects on SBS.

The objectives, so, in therapy for SBS, as have been mentioned above, are threefold: first, to keep the patient well nourished and growing proportionately; second, to keep fluid and electrolyte status stable, minimising the faecal loss of fluid, electrolytes and nutrients; and finally, to maximise the process of bowel adaptation.

The interaction, however, of bacterial overgrowth and the intestinal barrier seems to be of extreme importance in the SBS patient since the direct translocation of bacteria across the intestinal epithelium contributes to the genesis of the symptoms characterising this syndrome.

\section{Intestinal barrier-bacterial translocation interaction}

The intestinal barrier is the interface between the luminal contents of the gut and the intestinal mucosa that harbours the gut-associated immune system. A complex of several immunological and non-immunological factors maintains the barrier function of the gastrointestinal tract. The immune system of the intestine includes Peyer's plaques, lymphoid cells located within the intestinal lamina propria, intra-epithelial lymphocytes and aggregated lymphoid tissue within the mesenteric lymph nodes. In healthy individuals, it provides an effective barrier against luminal antigens and toxins.
An impairment of this intestinal barrier is associated with the pathogenesis of several diseases ${ }^{(26)}$. Translocation is used to describe the passage of viable resident bacteria from the gastrointestinal tract, across the mucosa, to normally sterile tissues such as the mesenteric lymph nodes and other internal organs ${ }^{(27)}$. The term also applies to the passage of inert particles and other antigenic macromolecules, such as lipopolysaccharide endotoxins and peptidoglycans, across the intestinal mucosal barrier ${ }^{(28)}$. It is usually assumed that the colon, with its much higher bacterial load, must be the most probable site of BT. It would seem unlikely that translocation occurs from other parts of the normally sterile intestinal tract but there is no clinical or experimental evidence to confirm this. It is tempting to think that any bacteria or endotoxin passing through the intestinal barrier might cause septic complications in the host, but there is growing evidence to suggest that translocation may in fact be a normal phenomenon ${ }^{(29)}$. It is possible that translocation occurs to allow the alimentary tract to be exposed to and sample antigens within the lumen such that the gut can mount a controlled local immune response helping to keep these antigens away from the internal milieu, a process known as 'oral tolerance $^{,(30)}$. It is then only when the host's immune defences are overwhelmed or otherwise defective that septic complications arise. Relationships between bacterial overgrowth and systemic sepsis are of potential importance in the intestinal failure patient because the direct translocation of bacteria across the intestinal epithelium may contribute to systemic sepsis.

Although the pathogenesis of sepsis and multiple organ failure in critical illness remains obscure, it is generally agreed that the gut plays a pivotal role ${ }^{(31)}$. The precise mechanism is unclear, but there is evidence to suggest that changes in gastrointestinal microflora associated with the use of antibiotics, immunosuppression or changes in intestinal permeability may disrupt the normal ecological balance and alter gut barrier function ${ }^{(32)}$. This may predispose to the translocation of bacteria or endotoxins, which may in turn result in systemic inflammation and septic morbidity. Bacteria carry toxins that can bind and activate Toll-like receptors (TLR), which stimulate, via a signalling cascade, the production of many pro-inflammatory cytokines, leading to inflammation and possible sepsis. The TLR have been implicated in many human disease states $^{(33)}$. The Kupffer cells in the liver are a major source of pro-inflammatory cytokines, which can lead to organ dysfunction. TLR4 seems to be the main lipopolysaccharide receptor, although co-receptors such as CD14 are also involved in regulating the hepatic inflammatory cascade. In addition to sepsis having been shown to decrease intestinal adaptation, many studies have examined the detrimental effects of pro-inflammatory cytokines on the liver ${ }^{(34)}$.

In critically ill patients, the balance of the gut microecology is altered in such a way that the number of potentially 
pathogenic bacteria increases and the healthy interaction between the host and microbes is disturbed. Strategies intended to protect the gut during critical illness have been predominately aimed at the maintenance of adequate splanchnic perfusion and prevention of mucosal ischaemia-reperfusion injury ${ }^{(35)}$. Another approach has been the use of nutritional therapies directed towards promoting enterocyte function, an example of which is glutamine ${ }^{(36)}$. One other potential target is the gastrointestinal microflora ${ }^{(37)}$. Commensal bacteria contribute substantially to the gut mucosal barrier through diverse means, including colonisation resistance, immunomodulation and mucus production.

In conclusion, the exact mechanism behind BT is still not known, but attachment of bacteria to the mucosal surface is a prerequisite in the pathogenesis of many infections originating from the gut. Also, for a probiotic bacterium to exert any biological effect, adherence to the mucosa seems to be of importance. However, it remains to be clarified whether adherence of a probiotic is a prerequisite for preventing $\mathrm{BT}$ of the indigenous microflora.

\section{Syn-, pre- and probiotics}

Probiotics are non-pathogenic bacteria, originally derived from the alimentary tract, which beneficially affect the host by improving the colonic microenvironment through fostering of colonisation. They may offer alternatives for the management of the growing problem of multiple antibiotic resistance and overwhelming infections in patients suffering different gastrointestinal disorders ${ }^{(38)}$. Various bacterial genera most commonly used in probiotic preparations are Lactobacillus, Bifidobacterium, and Escherichia coli, Enterococcus, Bacillus and Streptococcus. Some fungal strains belonging to Saccharomyces have also been used ${ }^{(38)}$. The beneficial effects of probiotics have been reported in several situations such as diarrhoea ${ }^{(39)}$, food allergies ${ }^{(40)}$, immune disorders ${ }^{(41)}$, inflammatory bowel disease ${ }^{(42)}$, prevention of the formation of intestinal tumours $^{(43)}$ and prevention of body-weight loss in animal models $^{(44)}$.

The positive effects of probiotic microbial species are mainly attributed to changing the composition of the gut microflora, by competition with pathogens for nutrients and mucosal adherence, by production of antimicrobial substances that modulate mucosal and systemic immune activity and epithelial function, by enhanced epithelial cell survival, and by improved immune responses ${ }^{(45,46)}$. In addition, probiotics metabolise nutrients into volatile fatty acids and chemically modified bile acids that create a local environment unfavourable for the growth of many intestinal pathogenic bacteria ${ }^{(47)}$. Probiotics produce large amounts of SCFA in the large intestine ${ }^{(48)}$. SCFA such as butyrate, propionate and acetate reportedly stimulate intestinal epithelial cell proliferation, intestinal mucin production, excretion of pancreatic enzymes, and intestinal motility, and decrease epithelial cell apoptosis ${ }^{(49)}$.

The potential and evidence-based mechanisms of action $^{(4)}$ by which probiotics can interact with the intestinal barrier and promote gastrointestinal health can be summarised to the following: probiotics and surface-layer proteins competitively exclude microbial pathogens from mucosal surfaces. Tight junction proteins, such as zona occludins1 and claudin-1, remain intact and thereby prevent both the uptake of intact macromolecules and translocation of viable organisms (BT) to the mesenteric lymph nodes, and ultimately to the liver. Through a cascade of signalling events, probiotics enhance the production and secretion of anti-inflammatory cytokines, including IL-10 and transforming growth factor- $\beta$, by a subset of immune cells, referred to as $\mathrm{T}$ regulatory cells. Innate immune responses to probiotics include increased mucin and trefoil factor production by goblet cells and enhanced production of antibacterial defensins by Paneth cells and intestinal epithelia. Probiotics might also alter the intestinal microbiota and hence limit intestinal bacteria overgrowth and the production of lipopolysaccharides.

In small animal models, several reports have suggested that probiotics enhance the local and systemic immune system through an increased activity of IgA, T-cells, macrophages, Th1-cytokines, as well as the modulation of gut-associated lymphoid tissue, and natural killer cell cytotoxicity $^{(50-55)}$.

Prebiotics, on the other hand, are short-chain carbohydrates (oligosaccharides) that have unusual effects in the gut. They alter the composition, or balance, of the microbiota, both in the lumen and at the mucosal surface, to one in which bifidobacteria and lactobacilli come to greater prominence. This, so-called healthier, flora should provide increased resistance to gut infections and may also have immunomodulatory properties. Prebiotics also act as carbon and energy sources for bacteria growing in the large bowel, where they are fermented to SCFA and are energy sources for the gut and other body tissues ${ }^{(56)}$. Additionally, they function as an alternative to probiotics, which can be difficult to handle in some foodstuffs, but whose benefits to health in terms of diarrhoea prevention and immunomodulation are becoming increasingly well established. Prebiotics currently in use, especially inulin and its derivatives, and galacto-oligosaccharides, are relatively cheap to manufacture or extract from plant sources, in addition to having beneficial effects on the gut microbiota and host. They are also valuable functional ingredients in foods with the potential to give fat-based spreads and dairy products improved organoleptic properties ${ }^{(57)}$. In a clinical context, prebiotics are relatively poor laxatives and have been used without much success to manage constipation.

Combined treatment with probiotics and prebiotics is called synbiotic therapy and is expected to have a stronger effect on intestinal diseases than probiotics or prebiotics alone. 
Probiotics seem to have broad beneficial effects, both as a food and as a therapeutic agent. There are specific identified mechanisms in some, but not all, of these bacteria that are important relative to the pathogenesis of bowel diseases. Several studies as mentioned, relative to the mechanism of action of probiotics, have identified that these organisms can have a direct effect on epithelial cell function and intestinal health. For probiotics to have a therapeutic role in the management of clinical SBS, their therapeutic mechanism of action must be aligned with the pathogenic mechanism of action of the disease.

There are currently no published full papers of randomised controlled trials concerning the use of prebiotics alone in SBS. The pathogenesis of SBS may preclude the use of prebiotics alone in this condition, as a well-known consequence of feeding even moderate amounts of some of the currently favoured prebiotics is increased gas production in the gut, because of their rapid fermentation in the proximal bowel $^{(57)}$. However, a number of studies using synbiotics have been carried out with varying results.

\section{Animal and experimental studies on short bowel syndrome and probiotics}

Recently, the effectiveness of probiotic, prebiotic and synbiotic therapy for the improvement of intestinal microbial balance ${ }^{(58)}$, prevention of $\mathrm{BT}^{(59)}$ and improvement of nutritional status in animal models ${ }^{(60)}$ with SBS has been reported.

Mogilner et al. ${ }^{(59)}$ in a rat SBS model for BT and intestinal integrity claimed that treatment with probiotics resulted in a significant decrease in BT and decreased enterocyte apoptosis compared with SBS untreated animals. SBS rats showed a significant increase $(v$. sham) in jejunal and ileal bowel and mucosal weight, mucosal DNA and protein, villus height and crypt depth. SBS rats also had a greater proliferation index and apoptotic index in both the jejunum and ileum compared with sham animals. SBS probiotic rats showed a significant increase ( $v$. SBS rats) in crypt depth in the ileum and a mild decrease in apoptotic index in the jejunum and ileum, compared with SBS untreated animals.

Eizaguirre et al. ${ }^{(60)}$ in another study tried to test the hypothesis that Bifidobacterium lactis administration decreases BT in SBS in animals fed orally. BT was detected in $6 \%$ of control group rats. The incidence of BT in the sham group was $87 \%$ (thirty-four of thirty-nine), whereas only $50 \%$ (nine of eighteen) of probiotic-treated animals had BT $(P<0.05)$. The relative risk reduction (RRR) was $0.43(95 \% \mathrm{CI} 0.14,0.72)$, and the number needed to treat (NNT) was 3 (95\% CI 2, 8). In other words, animals that received $B$. lactis had the risk of $\mathrm{BT}$ reduced by $43 \%$ (RRR of 0.43 ), and of every three animals treated, one is expected to be free of BT (NNT of 3).

Additionally, García-Urkia et al. ${ }^{(61)}$ demonstrated that administration of $B$. lactis reduces the incidence of BT in adult Wister rats, after $80 \%$ gut resection. At the end of the experiment mesenteric lymph nodes, and peripheral and portal blood specimens were recovered and cultured. BT was detected in 93\% of sham rats. The incidence of BT in probiotic-treated animals was $44 \%$.

Several reports in the international literature have tested and proved the preservation of tight junction protein expression, inhibition of epithelial apoptosis, decrease in pathogenic bacterial adhesion, reduction of pro-inflammatory cytokines, and increase in mucus production and defensin secretion; these are some of the mechanisms, namely, that are responsible for the intestinal barrier-preserving effect of probiotic bacteria ${ }^{(62-67)}$.

At this point, it may be valuable to refer to two indirectly related studies that deal with liver damage, sepsis, the intestinal barrier and probiotics in an effort to support the reverse hypothesis of the following sequence of phenomena: intestinal failure leading to BT, leading to potential sepsis, leading to hepatic damage. In the first study by Osman et al. it was demonstrated that probiotics effectively attenuate liver damage and maintain gut barrier and epithelial function in a microbial (D-galactosamine and lipopolysaccharide)-induced sepsis murine model ${ }^{(68)}$. Sprague-Dawley rats were used and separated in six experimental groups: acute liver injury control and five groups of liver injury treated by blueberry alone or by each of the probiotic strains (Lactobacillus plantarum DSM 15313 and B. infantis DSM 15159) with and without blueberry. Alanine aminotransferase levels decreased significantly in all groups compared with liver injury control and DSM 15313 groups. Bilirubin, liver TNF- $\alpha$, myeloperoxidase and acetic acid in caecum contents decreased significantly in all groups, while liver glutathione values increased significantly in all groups compared with the liver injury control group. Liver IL-1 $\beta$ and BT to the liver and mesenteric lymph nodes decreased significantly in all groups except the $B$. infantis DSM 15159 group compared with the liver injury control group. Enterobacteriaceae count in the caecum decreased significantly in the groups with blueberry plus probiotics compared with the other groups. The study's conclusion was that blueberry and probiotics exert protective effects on acute liver injury. They reduce hepatocyte injury, inflammation and proinflammatory cytokines, and improve barrier function and antioxidant activity.

Ewaschuk et al. ${ }^{(31)}$ with their study aimed to determine the efficacy of a probiotic compound in attenuating hepatic and intestinal injury in a mouse model of sepsis. Wild-type and IL-10 gene-deficient $129 \mathrm{~Sv} / \mathrm{Ev}$ mice were fed the probiotic compound VSL\#3 for $7 \mathrm{~d}$. To induce sepsis, the mice were injected with lipopolysaccharide and D-galactosamine in the presence and absence of the PPAR $\gamma$ inhibitor GW9662. The livers were analysed for BT, cytokine production, histological injury and PPAR $\gamma$ levels. Mice injected with lipopolysaccharide-D-galactosamine demonstrated a breakdown in colonic barrier function, which correlated 
with enhanced pro-inflammatory cytokine secretion, BT and significant hepatic injury. The authors' conclusion was that a pre-treatment with oral probiotics prevented the breakdown in intestinal barrier function, reduced BT and significantly attenuated liver injury. The inhibition of PPAR $\gamma$ with GW9662 abrogated the protection induced by probiotics.

Evidence from experimental animal studies, as has been previously reported, consistently indicates that probiotics exert barrier-enhancing, antibacterial, immune-modulating and anti-inflammatory effects, which all could be beneficial in small-intestinal bacterial overgrowth and intestinal failure.

\section{Clinical studies on short bowel syndrome and probiotics}

Long-term follow-up studies in nutrition assessment of paediatric patients with SBS, even after weaning off PN for a long time, proved that $\mathrm{PN}$ alone is not enough and that new combined treatment formulas are required ${ }^{(69)}$.

In human subjects, there are several reports concerning probiotic enhancement of the local immune system through the development of IgA secretion against gastrointestinal infections, modulation of human dendritic cell function, and enhancing the activity of natural killer cells $^{(70-72)}$. Moreover, other studies have demonstrated the efficacy of syn-, pre- and probiotic administration in clinical situations such as in critically ill patients ${ }^{(73)}$, in surgical wound infection ${ }^{(74)}$ and in SBS with refractory enterocolitis. After an extended search of the international literature, only a few clinical studies managed to be found referring to SBS and probiotics, with only two of them being randomised controlled trials.

The use of probiotics for the prevention of NEC and short gut in neonates has been documented by Alfaleh et $a .^{(75)}$ in nine eligible trials randomising 1425 infants. The trials included were highly variable with regard to enrolment criteria, baseline risk of NEC in the control groups, timing, dose, formulation of the probiotics, and feeding regimens. In a meta-analysis, enteral probiotic supplementation significantly reduced the incidence of severe NEC and mortality. There was no evidence of a significant reduction of nosocomial sepsis or days on total parenteral nutrition. The statistical test of heterogeneity for NEC, mortality and sepsis was insignificant. Data regarding extremely low-birth-weight infants could not be extrapolated. The included trials reported no systemic infection with the supplemental probiotic organism.

Kanamori et al. referred to their experience of long-term synbiotic therapy in two studies. In the first one ${ }^{(76)}$, seven short bowel patients with refractory enterocolitis were studied, suggesting that this protocol might be a potent modulator in intestinal flora, by increasing the SCFA and accelerating patients' body-weight gain. The authors designed a protocol for synbiotic therapy composed of B. breve, L. casei and galacto-oligosaccharides, and preliminarily ascertained its clinical effects in human subjects. This protocol of synbiotic therapy was applied for more than 1 year to seven malnourished patients with SBS who suffered from refractory enterocolitis. The therapeutic protocol resulted in improvement of the intestinal bacterial flora (inducing domination by anaerobic bacteria and suppressing the residence of pathogenic bacteria) and increased SCFA in the faeces (from 27.8 to $65.09 \mu \mathrm{mol} / \mathrm{g}$ wet faeces). All patients but one accelerated their body-weight gain, and five patients showed increased serum rapid turnover proteins.

The other study ${ }^{(77)}$ reported the use of synbiotic therapy in the treatment of a 4 -year-old girl suffering from SBS. For the synbiotic therapy, B. breve, L. casei and galacto-oligosaccharides were used. This novel combination therapy was expected to act synergistically for the improvement of the subject's health. The patient's intestinal absorptive function and motility were dramatically improved by this newly designed synbiotic therapy, and she progressed satisfactorily after 2 years of therapy.

Uchida et al. ${ }^{(78)}$ by administrating synbiotic therapy including $B$. breve, L. casei and galacto-oligosaccharides in four paediatric patients with SBS claimed an improvement in the immunonutritional status of the patients. Enrolled as controls were four normal, healthy, age-matched children. Faecal samples from patients and controls were collected and analysed for faecal bacterial flora and organic acid contents. Levels of SCFA such as butyrate, propionate and acetate increased in one patient, and SCFA and total organic acid levels increased in three patients. Serum lymphocyte counts and concentrations of pre-albumin increased after beginning synbiotic therapy, reaching a statistically significant level at the ninth month compared with the pre-treatment level. There was an increasing trend in height and weight gain velocity during the study compared with the pre-treatment period. The patients' faecal bacterial flora improved as a result of the synbiotic therapy.

Jain et $a{ }^{(79)}$ in a prospective and randomised trial suggested that the enteral administration of a synbiotic preparation to critically ill patients can significantly alter the microbial composition of the upper gastrointestinal tract. A total of ninety patients admitted to an intensive care unit were randomised to receive either synbiotic or placebo preparations (forty-five patients in each group). The synbiotic preparation consisted of L. acidophilus La5, B. lactis Bb 12, Streptococcus thermophilus and L. bulgaricus (probiotics) with oligofructose (prebiotic). Gut barrier function was assessed by the measurement of intestinal permeability (lactulose/rhamnose test) and by the culture of nasogastric aspirate on days 1 and 8 . All septic complications and mortality were recorded. The research resulted that there were no differences between the groups in terms of age, sex, APACHE II (Acute Physiology and Chronic Health Evaluation II) or POSSUM (Physiologic and Operative Severity Score for the enUmeration of Mortality and Morbidity) scores. After 1 week of therapy, patients in the synbiotic group had a significantly lower incidence of 
potentially pathogenic bacteria (43v.75\%; $P<0.05$ ) and multiple organisms (39v. 75\%; $P<0.01)$ in their nasogastric aspirates than controls. There were no significant differences between the groups in terms of intestinal permeability, septic complications or mortality. As a conclusion the high incidence of enteric organisms isolated simultaneously from nasogastric aspirates and septic foci in this study provides further circumstantial evidence in support of the gut-origin of sepsis hypothesis.

However, the fact that more clinical studies are required to ensure statistically important results is obvious. In one recent double-blind, placebo-controlled, cross-over trial the effects of supplementation with L. rhamnosus GG (LGG) on intestinal permeability in children with SBS were examined ${ }^{(80)}$. A total of twenty-one children (nine children with SBS and twelve healthy control children), aged between 1.6 and 16.4 years, participated in this cross-over study. Subjects with SBS received LGG (in the form of a capsule containing $10^{9}$ bacteria) or placebo (identical to the LGG capsule) for 4 weeks, followed by a 3-week washout before therapy was crossed over for another 4 weeks. Intestinal permeability, quantitative faecal cultures for Lactobacillus species (in colony-forming units/g stool) and the hydrogen breath test (HBT) were performed during the LGG and placebo phases of therapy. The results demonstrated that baseline intestinal permeability measurements were similar in patients with SBS and control subjects and that intestinal permeability was correlated with age in control subjects but not among patients with SBS. Furthermore, faecal colonisation with Lactobacillus species did not differ during LGG $v$. placebo therapy, respectively, and LGG therapy had no consistent effects on intestinal permeability or its relationship with age and was associated with conversion to positive HBT results in one subject. The authors concluded that in this sample of children with SBS, intestinal permeability was within normal limits but did not correlate with age and that LGG therapy had no consistent effects on intestinal permeability. These findings, finally, do not support empirical LGG therapy to enhance intestinal permeability in children with SBS, as there was no statistically significant difference in intestinal permeability between the two groups.

Probiotic agents are generally safe, but complications of their use can occur. Several reports describe Lactobacillus bacteraemia, D-lactic acidosis and liver abscess formation after probiotic treatment in patients with short gut syndrome ${ }^{(81)}$.

De Groote et $a l{ }^{(82)}$ described a case of bacteraemia after ingestion of a LGG probiotic tablet in a child with short gut syndrome. Sequencing of the ribosomal operon region and strain typing with pulsed field electrophoresis of the isolates showed identity between the tablet and bloodstream isolates.

In another study, Kunz et al. ${ }^{(83)}$ referred to two cases treated with LGG supplementation for short gut syndrome and demonstrated that this strain particularly when associated with intestinal friability may be responsible for bacteraemia or sepsis. In conclusion they suggested that these patients should be closely monitored for signs of sepsis, and empirical therapy for sepsis should include coverage of this organism pending specific culture results.

\section{Conclusion}

The accurate diagnosis of bacterial overgrowth and the SBS process presents a number of challenges in clinical practice and especially so among patients with intestinal failure. The current challenge, therefore, is to determine which medical or surgical therapies (if any) can be successfully applied in the complicated intestinal failure patient that can avoid the need for transplantation (thereby avoiding the risk for pretransplant waiting list mortality) and the long-term risks from immunosuppression and provoking simultaneously the autologous mechanism of intestinal adaptation.

Nowadays, orally given nutritive supplements tend to appear as the 'gold standard' to promote intestinal adaptation. One category of those seems to be probiotics, although the exact mechanism of their action in altering gastroenteric microflora is not clear. Both local and remote actions of probiotic organisms have been suggested. Some studies have shown a reduction in potentially pathogenic organisms even at sites distant to the gut (vagina and nasal cavity) following oral probiotic therapy. Overall, studies in in vitro systems and in a wide range of animal models provide considerable evidence that probiotics, prebiotics and synbiotics exert some protective effects. However, evidence from human studies is still limited. Furthermore, research is required to identify the probiotic, prebiotic or synbiotic combination that will be most effective for humans. It is very likely that there will not be an ideal treatment for all cases, but the treatment will depend on the each individual's unique intestinal flora composition.

New options are given through the genetic manipulation of probiotics, designed to act as a delivery system in the bowel. Although this field of study is promising and exciting, this enthusiasm should be tempered by the fact that we are probably many years away from determining how to use these agents and their ultimate role may remain quite limited.

Finally, based on the limited clinical data, we would like to emphasise the fact that researchers and clinicians should remain sceptical and critical when supplementing probiotics, especially as a therapy measure. There is certainly a need for more carefully designed human clinical trials using different preparations for more prolonged periods. SBS is a really complex pathophysiological entity and combination treatment should be the first priority in order to ensure a better prognosis and outcome for the patient.

\section{Acknowledgements}

The authors state that there was no extra-institutional funding. The study was supported by the National and Kapodistrian University of Athens. 
C. N. S., E. P. M., B. G. S. and P. P. made contributions to the conception and design of the study. C. N. S. contributed to the analysis and interpretation of data and wrote the manuscript. All authors read and approved the final manuscript. All authors contributed equally to the final draft of the manuscript. C. I. F. gave the final approval of the version to be published.

The authors declare that they have no competing interests.

\section{References}

1. Misiakos EP, Macheras A, Kapetanakis T, et al. (2007) Short bowel syndrome: current medical and surgical trends. $J$ Clin Gastroenterol 41, 5-18.

2. Spencer AU, Neaga A, West B, et al. (2005) Pediatric short bowel syndrome: redefining predictors of success. Ann Surg 242, 403-408.

3. Quirós-Tejeira RE, Ament ME, Reyen L, et al. (2004) Longterm parenteral nutritional support and intestinal adaptation in children with short bowel syndrome: a 25 -year experience. J Pediatr 145, 157-163.

4. Gratz SW, Mykkanen H \& El-Nezami HS (2010) Probiotics and gut health: a special focus on liver diseases. World J Gastroenterol 16, 403-410.

5. Gonzalez HF, Perez NB, Malpeli A, et al. (2003) Nutrition and immunological status in long-term follow up of children with short bowel syndrome. J Parenter Enter Nutr 29, 186-191.

6. Goday PS (2009) Short bowel syndrome: how short is too short? Clin Perinatol 36, 101-110.

7. Cassaccia G, Trucchi A, Spirydakis I, et al. (2006) Congenital intestinal anomalies, neonatal short bowel syndrome, and prenatal counseling. J Pediatr Surg 41, 804-807.

8. Sigalet DL (2001) Short bowel syndrome in infants and children: an overview. Semin Pediatr Surg 10, 49-55.

9. O'Keefe SJ, Buchman AL, Fischbein TM, et al. (2006) Short bowel syndrome and intestinal failure: consensus definitions and overview. Clin Gastroenterol Hepatol 4, 6-10.

10. Wessel JJ \& Kocoshis SA (2007) Nutritional management of infants with short bowel syndrome. Semin Perinatol 31, 104-111.

11. Goulet O, Baglin-Gobet S, Talbotec C, et al. (2005) Outcome and long term growth after extensive small bowel resection in the neonatal period: a survey of 87 children. Eur J Pediatr Surg 15, 95-101.

12. DiBaise JK, Young RJ \& Vanderhoof JA (2004) Intestinal rehabilitation and the short bowel syndrome. Am J Gastroenterol 99, 1386-1395.

13. Juno RJ, Knott AW, Profitt SA, et al. (2004) Preventing enterocyte apoptosis after massive small bowel resection does not enhance adaptation of the intestinal mucosa. J Pediatr Surg 39, 907-911.

14. Folkman J (2007) Is angiogenesis an organizing principle in biology and medicine? I Pediatr Surg 42, 1-11.

15. Lee KD, Yamataka A, Kato Y, et al. (2006) Basic fibroblast growth factor and granulocyte colony-stimulating factor enhance mucosal surface expansion after adult small bowel transplantation without vascular reconstruction in rats. J Pediatr Surg 41, 737-741.

16. Parvadia JK, Keswani SG, Vaikunth S, et al. (2007) Role of VEGF in small bowel adaptation after resection: the adaptive response is angiogenesis dependent. Am J Physiol Gastrointest Liver Physiol 293, G591-G598.
17. Martin CA, Perrone EE, Longshore SW, et al. (2009) Intestinal resection induces angiogenesis within adapting intestinal villi. J Pediatr Surg 44, 1077-1083.

18. Murakami M, Sato N, Sato N, et al. (2007) Changes in lymphocyte phenotypes and cytokine production by surgical stress in a rat small intestinal resection model.J Clin Biochem Nutr 40, 216-220.

19. Diamanti A, Basso MS, Castro M, et al. (2008) Irreversible intestinal failure: prevalence and prognostic factors. J Pediatr Gastroenterol Nutr 47, 450-457.

20. Duro D, Kamin D \& Duggan C (2008) Overview of pediatric short bowel syndrome. J Pediatr Gastroenterol Nutr $\mathbf{4 7}$, S33-S36.

21. Diamanti A, Basso MS, Castro M, et al. (2007) Prevalence of life-threatening complications in pediatric patients affected by intestinal failure. Transplant Proc 39, 1632-1633.

22. Nightingale JM (2003) Hepatobiliary, renal and bone complications of intestinal failure. Best Pract Res Clin Gastroenterol 17, 907-929.

23. Atalay F, Ozcay N, Gundogdu H, et al. (2003) Evaluation of the outcomes of short bowel syndrome and indications for intestinal transplantation. Transplant Proc 35, 3054-3056.

24. Goulet O \& Ruemmele F (2006) Causes and management of intestinal failure in children. Gastroenterology 130, S16-S28.

25. Sukhotnik I, Coran AG, Alexander Kramer A, et al. (2005) Advances in short bowel syndrome: an updated review. Pediatr Surg Int 21, 947-953.

26. Clayburgh DR, Shen L \& Turner JR (2004) A porous defense: the leaky epithelial barrier in intestinal disease. Lab Invest 84, 282-291.

27. Quigley EMM \& Quera R (2006) Small intestinal bacterial overgrowth: roles of antibiotics, prebiotics, and probiotics. Gastroenterology 130, S78-S90.

28. Resta-Lenert S \& Barrett KE (2003) Live probiotics protect intestinal epithelial cells from the effects of infection with enteroinvasive Escherichia coli (EIEC). Gut 52, 988-997.

29. Gatt M, Reddy BS \& Macfie J (2007) Review Article: bacterial translocation in the critically ill - evidence and methods of prevention. Aliment Pharmacol Ther 25, 741-757.

30. O'Brien DP, Nelson LA, Kemp CJ, et al. (2002) Intestinal permeability and bacterial translocation are uncoupled after small bowel resection. I Pediatr Surg 37, 390-394.

31. Ewaschuk J, Endersby R, Thiel D, et al. (2007) Probiotic bacteria prevent hepatic damage and maintain colonic barrier function in a mouse model of sepsis. Hepatology $\mathbf{4 6}$, 841-850.

32. Qin HL, Shen TY, Gao ZG, et al. (2005) Effect of Lactobacillus on the gut microflora and barrier function of the rats with abdominal infection. World J Gastroenterol 11, 2591-2596.

33. Cario E, Gerken G \& Podolsky DK (2007) Toll-like receptor 2 controls mucosal inflammation by regulating epithelial barrier function. Gastroenterology 132, 1359-1374.

34. Bruewer M, Luegering A, Kucharzik T, et al. (2003) Proinflammatory cytokines disrupt epithelial barrier function by apoptosis-independent mechanisms. J Immunol 171, 6164-6172.

35. Zareie M, Johnson-Henry K, Jury J, et al. (2006) Probiotics prevent bacterial translocation and improve intestinal barrier function in rats following chronic psychological stress. Gut 55, 1553-1560.

36. Spindler-Vesel A, Bengmark S, Vovk I, et al. (2007) Synbiotics, prebiotics, glutamine, or peptide in early enteral nutrition: a randomized study in trauma patients. J Parenter Enteral Nutr 31, 119-126.

37. Caballero-Franco C, Keller K, De Simone C, et al. (2007) The VSL\#3 probiotic formula induces mucin gene expression and 
secretion in colonic epithelial cells. Am J Physiol Gastrointest Liver Physiol 292, G315-G322.

38. Schrezenmeir J \& De Vrese M (2001) Probiotics, prebiotics and synbiotics - approaching a definition. Am J Clin Nutr 73, 361-364

39. De Vrese M (2003) Effects of probiotic bacteria on gastrointestinal symptoms, Helicobacter pylori activity and antibiotics-induced diarrhea. Gastroenterology 124, A560.

40. Pochard P, Gosset P, Grangette C, et al. (2002) Lactic acid bacteria inhibit TH2 cytokine production by mononuclear cells from allergic patients. J Allergy Clin Immunol 110, $617-623$.

41. Nagao F, Nakayama M, Muto T, et al. (2000) Effects of a fermented milk drink containing Lactobacillus casei strain Shirota on the immune system in healthy human subjects. Biosci Biotechnol Biochem 64, 2706-2708.

42. Mach T (2006) Clinical usefulness of probiotics in inflammatory bowel diseases. J Physiol Pharmacol 57, 23-33.

43. De Roos N \& Katan M (2000) Effects of probiotic bacteria on diarrhea, lipid metabolism, and carcinogenesis: a review of papers published between 1988 and 1998. Am J Clin Nutr 71, 405-411.

44. Bowen JM, Stringer AM, Gibson RJ, et al. (2007) VSL\#3 probiotic treatment reduces chemotherapy-induced diarrhea and weight loss. Cancer Biol Ther 6, 1449-1454.

45. Shanahan F (2002) The host-microbe interface within the gut. Best Pract Res Clin Gastroenterol 16, 915-931.

46. Guarner F \& Malagelada JR (2003) Gut flora in health and disease. Lancet 361, 512-519.

47. Tappenden KA \& Deutsch AS (2007) The physiological relevance of the intestinal microbiota - contributions to human health. J Am Coll Nutr 26, 679S-683S.

48. Sakata T (1987) Stimulatory effect of short-chain fatty acids on epithelial cell proliferation in the rat intestine: a possible explanation for trophic effects of fermentable fibre, gut microbes and luminal tropic factors. $\mathrm{Br} J \mathrm{Nutr}$ 58, $96-103$.

49. Katoh K (1995) Effects of short-chain fatty acids on exocrine and endocrine pancreatic secretion. In Physiological and Clinical Aspects of Short-Chain Fatty Acids, pp. 222-242 [J Cummings, J Rombeau and T Sakata, editors]. Cambridge: Cambridge University Press.

50. Taweechotipatr M, Iyer C, Spinler JK, et al. (2009) Lactobacillus saerimneri and Lactobacillus ruminis: novel human-derived probiotic strains with immunomodulatory activities. FEMS Microbiol Lett 293, 65-72.

51. Kimoto H, Mizumachi K, Okamoto T, et al. (2004) New Lactococcus strain with immunomodulatory activity: enhancement of Th1-type immune response. Microbiol Immunol 48, 75-82.

52. Roller M, Pietro Femia A, Caderni G, et al. (2004) Intestinal immunity of rats with colon cancer is modulated by oligofructose-enriched inulin combined with Lactobacillus rhamnosus and Bifidobacterium lactis. Br J Nutr 92, 931-938.

53. Matsuzaki T \& Chin J (2000) Modulating immune responses with probiotic bacteria. Immunol Cell Biol 78, 67-73.

54. Benyacoub J, Czarnecki-Maulden GL, Cavadini C, et al. (2003) Supplementation of food with Enterococcus faecium (SF68) stimulates immune functions in young dogs. J Nutr 133, 1158-1162.

55. Baillon ML, Marshall-Jones ZV \& Butterwick RF (2004) Effects of probiotic Lactobacillus acidophilus strain DSM13241 in healthy adult dogs. Am J Vet Res 65, 338-343.

56. Gibson GR, Probert HM, Van Loo J, et al. (2004) Dietary modulation of the human colonic microbiota: updating the concept of prebiotics. Nutr Res Rev 17, 259-275.
57. Macfarlane S, Macfarlane GT \& Cummings JH (2006) Review article: prebiotics in the gastrointestinal tract. Aliment Pharmacol Ther 24, 701-714.

58. Herbert MK \& Holzer P (2008) Standardized concept for the treatment of gastrointestinal dysmotility in critically ill patients - current status and future options. Clin Nutr 27, 25-41.

59. Mogilner JG, Srugo I, Lurie M, et al. (2007) Effect of probiotics on intestinal regrowth and bacterial translocation after massive small bowel resection in a rat. J Pediatr Surg $\mathbf{4 2}$, 1365-1371.

60. Eizaguirre I, Urkia NG, Asensio AB, et al. (2002) Probiotic supplementation reduces the risk of bacterial translocation in experimental short bowel syndrome. J Pediatr Surg 37, 699-702.

61. García-Urkia N, Asensio AB, Zubillaga Azpiroz I, et al. (2002) Beneficial effects of Bifidobacterium lactis in the prevention of bacterial translocation in experimental short bowel syndrome (article in Spanish). Cir Pediatr 15, 162-165.

62. Peuranen S, Tiihonen K, Apajalahti J, et al. (2004) Combination of polydextrose and lactitol affects microbial ecosystem and immune responses in rat gastrointestinal tract. Br J Nutr 91, 905-914.

63. Zyrek AA, Cichon C, Helms S, et al. (2007) Molecular mechanisms underlying the probiotic effects of Escherichia coli Nissle 1917 involve ZO-2 and $\mathrm{PKC} \zeta$ redistribution resulting in tight junction and epithelial barrier repair. Cell Microbiol 9, 804-816.

64. Yan F \& Polk DB (2002) Probiotic bacterium prevents cytokine-induced apoptosis in intestinal epithelial cells. $J$ Biol Chem 277, 50959-50965.

65. Schlee M, Harder J, Koeten B, et al. (2008) Probiotic lactobacilli and VSL $\# 3$ induce enterocyte $\beta$-defensin 2. Clin Exp Immunol 151, 528-535.

66. Madsen K, Cornish A, Soper P, et al. (2001) Probiotic bacteria enhance murine and human intestinal epithelial barrier function. Gastroenterology 121, 580-591.

67. Resta-Lenert S \& Barrett K (2009) Modulation of intestinal barrier properties by probiotics: role in reversing colitis. Ann N Y Acad Sci 1165, 175-182.

68. Osman N, Adawi D, Ahrne S, et al. (2007) Endotoxin- and D-galactosamine-induced liver injury improved by the administration of Lactobacillus, Bifidobacterium and blueberry. Dig Liv Dis 39, 849-856.

69. Wu J, Tang Q, Feng Y, et al. (2007) Nutrition assessment in children with short bowel syndrome weaned off parenteral nutrition: a long-term follow-up study. J Pediatr Surg 42, $1372-1376$.

70. Fukushima Y, Kawata Y, Hara H, et al. (1998) Effect of a probiotic formula on intestinal immunoglobulin A production in healthy children. Int J Food Microbiol 30, 39-44.

71. Mohamadzadeh M, Olson S, Kalina WV, et al. (2005) Lactobacilli activate human dendritic cells that skew T-cells toward T helper 1 polarization. Proc Natl Acad Sci US A 22, 2880-2885.

72. McNaught CE, Woodcock NP, MacFie J, et al. (2002) A prospective randomised study of the probiotic Lactobacillus plantarum $299 \mathrm{~V}$ on indices of gut barrier function in elective surgical patients. Gut 51, 827-831.

73. McNaught CE, Woodcock NP, Anderson AD, et al. (2005) A prospective randomised trial of probiotics in critically ill patients. Clin Nutr 24, 211-219.

74. Howard JC, Reid G \& Gan BS (2004) Probiotics in surgical wound infections: current status. Clin Invest Med 27, $274-281$

75. Alfaleh K, Anabrees J \& Bassler D (2009) Probiotics reduce the risk of necrotizing enterocolitis in preterm infants: a meta-analysis. Neonatology 97, 93-99. 
76. Kanamori Y, Sugiyama M, Hashizume K, et al. (2004) Experience of long-term synbiotic therapy in seven short bowel patients with refractory enterocolitis. J Pediatr Surg 39, 1686-1692.

77. Kanamori Y, Hashizume K, Sugiyama M, et al. (2001) Combination therapy with Bifidobacterium breve, Lactobacillus case $i$, and galactooligosaccharides dramatically improved the intestinal function in a girl with short bowel syndrome: a novel synbiotics therapy for intestinal failure. Dig Dis Sci 46, 2010-2016.

78. Uchida $\mathrm{K}$, Takahashi $\mathrm{T}$, Inoue $\mathrm{M}$, et al. (2007) Immunonutritional effects during synbiotics therapy in pediatric patients with short bowel syndrome. Pediatr Surg Int 23, 243-248.

79. Jain PK, McNaught CE, Anderson ADG, et al. (2004) Influence of synbiotic containing Lactobacillus acidophilus La5, Bifidobacterium lactis $\mathrm{Bb} 12$, Streptococcus thermophilus, Lactobacillus bulgaricus and oligofructose on gut barrier function and sepsis in critically ill patients: a randomised controlled trial. Clin Nutr 23, 467-475.

80. Sentongo TA, Cohran V, Korff S, et al. (2008) Intestinal permeability and effects of Lactobacillus rhamnosus therapy in children with short bowel syndrome. J Pediatr Gastroenterol Nutr 46, 41-47.

81. Snydman DR (2008) The safety of probiotics. Clin Infect Dis 46, S104-S111, discussion S144-S151.

82. De Groote MA, Frank DN, Dowell E, et al. (2005) Lactobacillus rhamnosus GG bacteremia associated with probiotic use in a child with short gut syndrome. Pediatr Infect Dis J 24, 278-280.

83. Kunz AN, Noel JM \& Fairchok MP (2004) Two cases of Lactobacillus bacteremia during probiotic treatment of short gut syndrome. J Pediatr Gastroenterol Nutr 38, 457-458. 